\title{
Circulating Tumor DNA in Saliva in an Orthotopic Head and Neck Cancer Mouse Model
}

\author{
YOUNG-GYU EUN ${ }^{1}$, YU JIN YOON ${ }^{1}$, KYU YEOUN WON $^{2}$ and YOUNG CHAN LEE ${ }^{1}$ \\ ${ }^{I}$ Department of Otolaryngology-Head \& Neck Surgery, School of Medicine, \\ Kyung Hee University, Seoul, Republic of Korea; \\ ${ }^{2}$ Department of Pathology, School of Medicine, Kyung Hee University, Seoul, Republic of Korea
}

\begin{abstract}
Aim: To evaluate the feasibility of conducting studies of saliva circulating tumor DNA (ctDNA) as a biomarker of metastasis or recurrence in our orthotopic head and neck cancer (HNC) mouse model. Materials and Methods: A mouse model of recurrence or metastasis after tongue cancer resection was developed. Blood and saliva were collected at baseline and at the establishment of recurrence or metastasis. Real-time polymerase chain reaction was performed to quantify human long interspersed element (hLINE) in samples to assess the amount of ctDNA. Results: In our model, salivary hLINE increased as the cancer developed and decreased after surgery. Plasma hLINE was significantly elevated in mice with metastasis. The presence of tongue cancer recurrence in mice was more correlated with hLINE concentration in saliva than in plasma. Conclusion: In our orthotopic model, salivary ctDNA better reflected tumor development and recurrence than did plasma ctDNA.
\end{abstract}

Head and neck cancer (HNC) is among the 10 most common cancer types worldwide. Diagnosis and monitoring response to therapy after treatment of patients with $\mathrm{HNC}$ can be performed by physical examination, as well as endoscopy and imaging [e.g. computed tomography (CT), magnetic resonance imaging (MRI) or positron-emission tomography (PET)]. However, $\mathrm{HNC}$ is usually difficult to diagnose early because initial lesions may be painless and abnormal epithelium can be hard to detect. Furthermore, fibrosis and inflammation of tissues after radiation therapy or surgery may cause failure in

Correspondence to: Young Chan Lee, MD, Ph.D., Department of Otolaryngology-Head and Neck Surgery, Kyung Hee University School of Medicine, \#892 Dongnamro, Gangdong-gu, Seoul 05278, Republic of Korea. Tel: +82 24406257, Fax: +82 24406296, e-mail: medchan@hanmail.net

Key Words: Head and neck cancer, saliva, recurrence, metastasis, circulating DNA, animal model. early diagnosis of residual cancer or recurrence by conventional endoscopy or imaging $(1,2)$. Although early detection and diagnosis lead to a greater survival rate and play a significant role in successful treatment, delayed diagnosis of disease or recurrence contributes to the high morbidity rate of HNC $(3,4)$. The current gold standard for HNC diagnosis is a biopsy; however, biopsy for suspicious lesions is limited by increased patient morbidity, the difficulty of finding suspected lesions and high costs.

To overcome these limitations, researchers have conducted a number of studies to develop effective and less invasive diagnostic methods for early-stage or recurrent HNC. Due to the nature of the anatomical location, the research and use of saliva-based diagnostics in $\mathrm{HNC}$ has increased exponentially over the years (5). However, there are currently no reliable diagnostic salivary biomarkers.

Circulating tumor DNA (ctDNA) is released by tumor cells into the blood and thus harbors mutations found in the original tumor (6). Therefore, ctDNA analysis might not only improve current systems of tumor diagnosis and facilitate early-stage detection, but also help determine tumor progression and prognosis, and assist in targeted therapy. Unfortunately, there has been little progress in understanding salivary ctDNA biology in HNC. In previous studies, an orthotopic mouse model using the tongue for induction of cancer was used to demonstrate the development of primary tumors with lymph node metastasis (7). This model can dynamically reflect biological changes caused by cancer development and metastasis. The concentration of human DNA sequences of long interspersed element-1 (LINE1), a member of the retrotransposon family, can be evaluated by quantitative real-time polymerase chain reaction (qPCR). This assay has been applied to measure human tumor cells in solid organs of animals and to measure the amount of ctDNA in mice harboring human cancer xenografts (8). Therefore, quantification of human DNA sequences in the saliva or plasma of a mouse model might also reflect ctDNA in saliva or plasma. 
Here, we assessed LINE1 concentrations in saliva and plasma in an orthotopic mouse model in order to evaluate the utility of our orthotopic HNC mouse model in conducting research into saliva ctDNA as a biomarker of metastasis or recurrence.

\section{Materials and Methods}

Tumor cell line. Human head and neck squamous cell carcinoma cell line FaDu (KCLB30043) was used for this study and was obtained from the Korean Cell Line Bank (Cancer research, Seoul National University, Seoul, Korea). Cells were cultured in Eagle's minimal essential medium (Biowest, Riverside, MO, USA) supplemented with $10 \%$ fetal bovine serum (Biowest) and $1 \%$ penicillin/streptomycin (Corning, NY, USA) at $37^{\circ} \mathrm{C}$ with $5 \% \mathrm{CO}_{2}$.

Orthotopic head and neck cancer animal model. All animal experiments were performed in compliance with the guidelines of the Animal Research Ethics Committee of Kyung Hee University and the Institutional Animal Care and Use committee of Kyung Hee University Hospital at Gangdong, Seoul, Republic of Korea (approval number: KHMC-IACUC-18-012). Five-week-old female BALB/cSlc-nu were purchased from Central Lab. Animal Inc. (Seoul, Republic of Korea) and acclimated for a week under specific-pathogen-free conditions. Mice were anesthetized with 25 $\mathrm{mg} / \mathrm{kg}$ of Zoletil 50 (Virbac, Carros, France) and $10 \mathrm{mg} / \mathrm{kg}$ of Rompun (Bayer Korea, Seoul, Republic of Korea) by intramuscular injection. A $0.25 \%$ trypsin solution (Gibco, Grand Island, NY, USA) was used to harvest FaDu cells. Trypsinized cells were washed twice and suspended in Dulbecco's phosphate-buffered saline (Biowest, Riverside, MO, USA) for injection. One million cells in $20 \mu$ Dulbecco's phosphate-buffered saline (DPBS) were transplanted by syringe with a $31-\mathrm{G}$ needle at the lateral border of the tongue of six-week-old BALB/cSlc-nu. Control mice were similarly injected with $20 \mu \mathrm{l}$ of DPBS only. The primary tumors of the tongue were excised 14 days after injection using a binocular microscope. Mice were sacrificed 3, 7, 14, 21, 35, 42, or 56 days after injection. Primary tumors, tongues and lymph nodes were collected after sacrifice and cut with scissors into two pieces; one was stored at $-80^{\circ} \mathrm{C}$ and the other was fixed in $10 \%$ neutralbuffered formalin for pathological analysis (Sigma-Aldrich, St Louis, MO, USA).

Tissue histopathology. All tumor and lymph node tissues were fixed in $10 \%$ neutral-buffered formalin and embedded in paraffin blocks. Four-micrometer sections of tissue were stained routinely with hematoxylin and eosin. The apoptosis index was calculated as the average number of apoptotic cells per 10 high-power fields (9).

Saliva and plasma collection for quantifying of ctDNA. Saliva and plasma were collected at serial time points following tumor induction. For collecting saliva, nude mice were anesthetized with $25 \mathrm{mg} / \mathrm{kg}$ wt of Zoletil 50 and $10 \mathrm{mg} / \mathrm{kg}$ wt of Rompun. Saliva secretion was induced by subcutaneously injecting $1.25 \mathrm{mg} / \mathrm{kg} \mathrm{wt}$ of pilocarpine and the dripping saliva was collected in sterilized 1.5$\mathrm{ml}$ tubes. Saliva was collected with natural flow while avoiding contamination by manipulation of surrounding tissues. Blood was collected by cardiac puncture with a heparin-(Sigma-Aldrich, MO, USA) coated syringe with a $26-\mathrm{G}$ needle. Mouse plasma samples were isolated through a two-step centrifugation process. Firstly, whole blood samples were centrifuged at $1,000 \times g$ at $4^{\circ} \mathrm{C}$ for 20 min. The supernatants were placed in new sterile $1.5 \mathrm{ml}$ tubes and then centrifuged at $3,000 \times g$ at $4^{\circ} \mathrm{C}$ for $10 \mathrm{~min}$.

LINE1 quantification by $q P C R$. DNA extraction was performed immediately after collection to prevent degradation of the ctDNA in mouse saliva and plasma using QIAamp DNA mini kit (Qiagen, Hilden, Germany) according to the protocol recommended by the manufacturer. Human-specific primers were used for detection of human LINE1 retrotransposon (sequences: forward 5'-TCACTCAAA GCCGCTCAACTAC-3', reverse 5'-TCTGCCTTCATTTCGTTATGT ACC-3'), generating an amplicon of 81 base pairs. Human LINE1 qPCR was carried out in duplicate using 2x SyGreen Mix Hi-ROX (qPCRBIO, London, UK) and the reaction was monitored on an ABI StepOnePlus Cycler (Applied Biosystems, Foster City, CA, USA). The reaction system was $20 \mu \mathrm{l}$, including $10 \mu \mathrm{L}$ SyGreen Mix Hi-ROX (2x), $1 \mu \mathrm{l}$ of PCR forward primer $(10 \mu \mathrm{M}), 1 \mu \mathrm{l}$ of PCR reverse primer $(10 \mu \mathrm{M}), 1 \mu \mathrm{l}$ of DNA template and $7 \mu \mathrm{l}$ of nuclease-free water. Tenfold serial dilutions of DNA purified from the FaDu cell line were incorporated in each plate to serve as standards. A no-template control (NTC; water) was included in every run. The threshold cycles (Ct) of NTC ranged from 31.9 to 34.4 and were considered as the effective laboratory background. Therefore, in this study, $\mathrm{Ct}$ values in the range of 31.9-34.4 were regarded as no amplification and represented as zero for analysis.

Statistical analysis. LINE1 concentration in saliva and plasma was log-transformed for analysis. A bivariate correlation assay was adopted for correlation analysis. The Mann-Whitney $U$-test or Student's $t$-test of two independent samples was applied to compare differences between two groups. One-way ANOVA was performed to compare the means among groups. All analyses were conducted using R statistical software (version R-3.6.0).

\section{Results}

Change in LINE1 concentration in saliva and plasma after tumor cell injection. In order to establish an orthotopic HNC model, we injected FaDu cells into the lateral border of the mouse tongue. After tumor cell injection, tongue cancer developed in $82.6 \%$ of mice (38/45) (Figure 1A). Within 1236 days after injection, tongue tumors from 18 mice were microsurgically removed (Figure 1B). After excision of tongue tumors, we observed recurrence in tongues of seven mice and regional metastasis in seven mice (Figure 1C and D). Saliva and plasma of four mice were collected at the time of no recurrence or metastasis after surgery. Figure 2 shows the change of LINE concentration in saliva and plasma through the days after $\mathrm{FaDu}$ cell injection. Two weeks after injection, when cancer was found in the tongues of most mice, LINE1 concentration in the saliva was the highest; concentration decreased rapidly after surgery. The concentration of LINE1 in the saliva increased again in mice where tongue cancer recurred. The concentration of LINE1 in the plasma also rose in mice with metastatic lesions. The change of LINE1 concentration in the plasma was different 

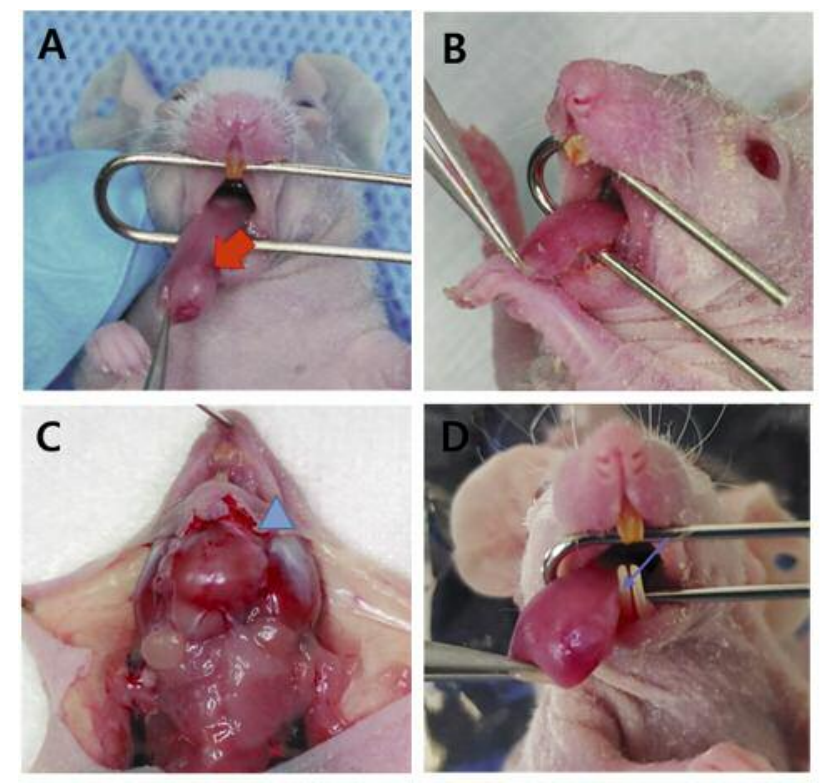

Figure 1. Establishment of an orthotopic head and neck cancer animal model. A: Primary tongue tumor growth in a representative orthotopic mouse 2 weeks after injection of FaDu cells. B: Representative mouse without tongue cancer after surgery. C: Representative mouse with cervical metastasis after surgery. D: Representative mouse with local recurrence after surgery.

from that in the saliva. The change in concentration of LINE1 in plasma was not as significant as in saliva, but increased with the development of tongue cancer. There was no significant decrease after surgery, but a rapid increase was observed when metastasis occurred. Compared with plasma, salivary LINE1 concentration more dynamically reflected tumor status and recurrence. However, LINE1 concentration in the plasma was more sensitive in reflecting metastasis.

LINE1 DNA quantification in saliva and plasma according to tumor status, recurrence, and metastasis. Salivary LINE1 DNA concentration was significantly correlated with plasma LINE1 DNA concentration in the orthotopic mouse model $(\mathrm{r}=0.48, p=0.001)$ (Figure 3$)$. We compared salivary and plasma LINE1 concentration according to the presence of tongue tumor (Figure 4). The LINE1 concentration in saliva was significantly increased in the presence of a tumor in the mouse tongue $(p=0.045)$. Although plasma LINE1 concentration increased with tumors compared to plasma of mice without a tumor, the difference was not statistically significant $(p=0.218)$. We analyzed the concentration of LINE1 according to recurrence of tongue tumor to determine whether ctDNA changes in saliva and plasma were relevant at the time of recurrence (Figure 5). When the tumor recurred, the salivary LINE1 concentration significantly

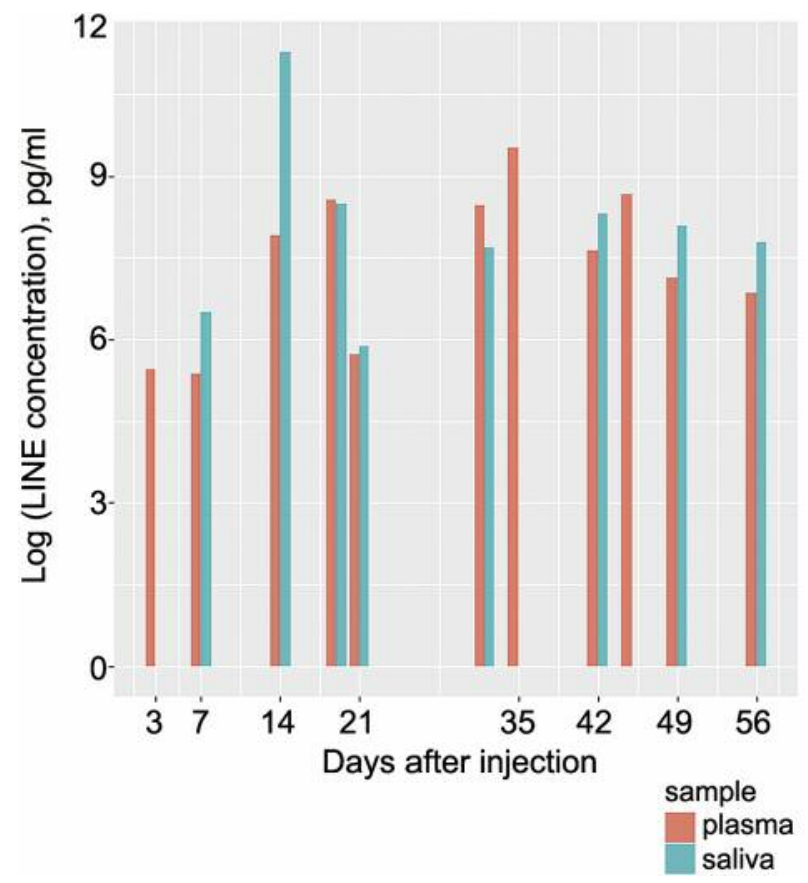

Figure 2. Time course of long interspersed element-1 (LINE1) concentration in saliva and plasma by day after injection of human head and neck squamous cell carcinoma cell line FaDu into mice.

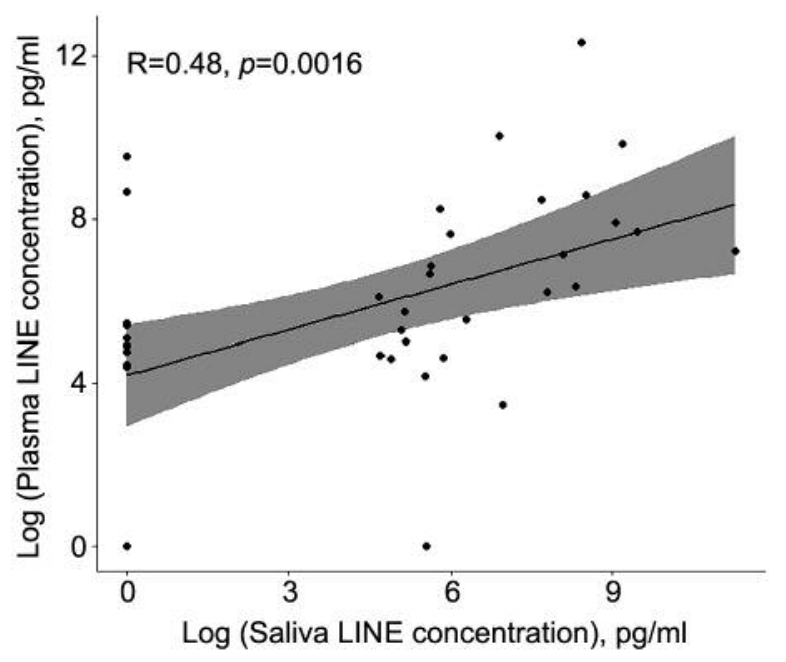

Figure 3. Correlation between long interspersed element-1 (LINE1) DNA concentrations in saliva and plasma.

increased $(p=0.048)$. There was no significant difference in plasma LINE1 concentration. We further investigated whether the LINE1 ctDNA concentration of saliva and plasma changed with metastasis of tongue tumors (Figure 6). 

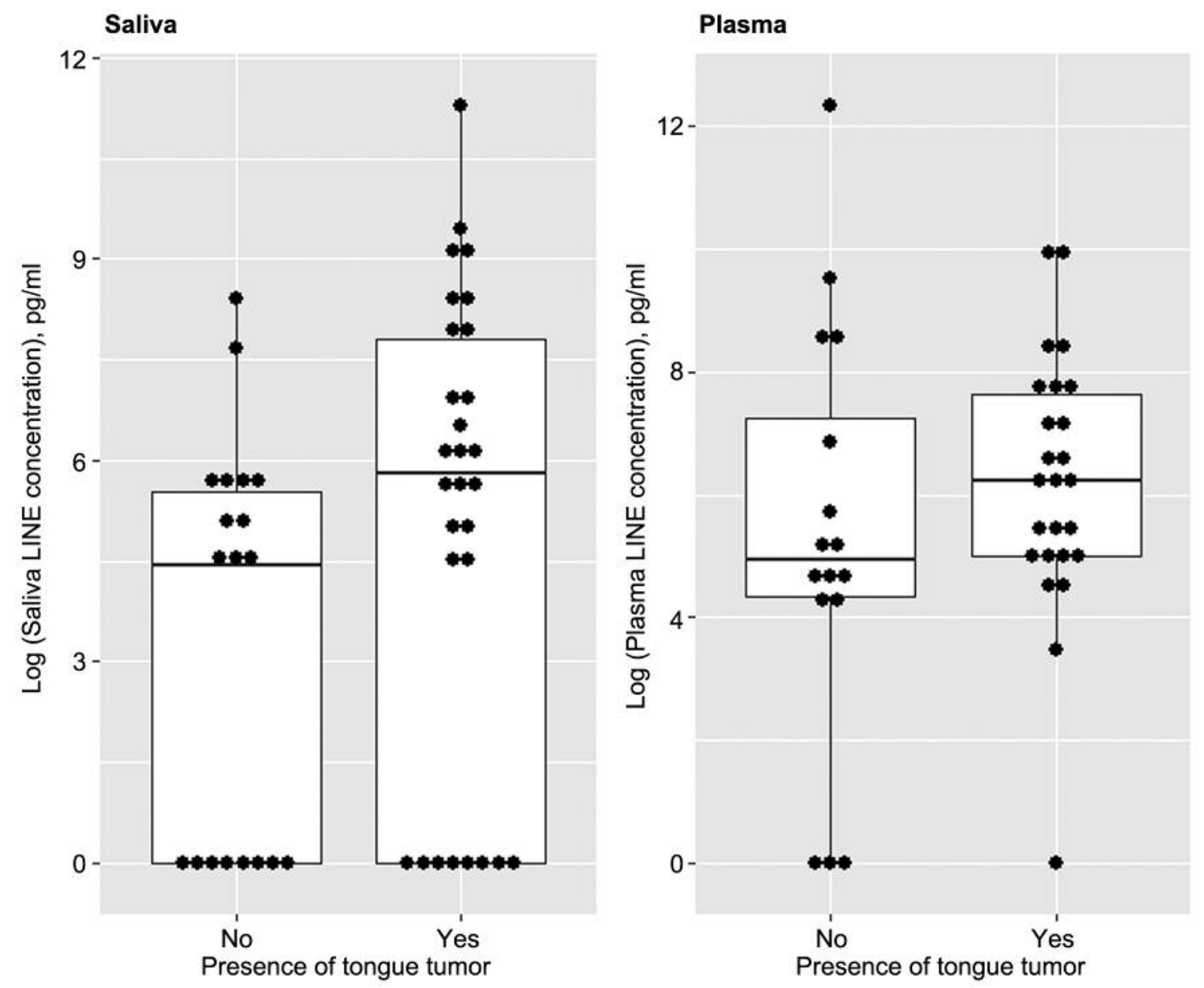

Figure 4. Comparison of long interspersed element-1 (LINE1) concentration in saliva and plasma according to the presence of tongue tumor.

Mouse tongues were free of lesions after surgery, but the LINE1 concentration in plasma was significantly higher when metastasis was observed $(p<0.001)$. However, salivary LINE1 concentration did significantly increase in the presence of metastasis. The concentration of LINE1 in saliva and plasma was compared between the following groups: i) control, ii) by primary tongue tumor status, iii) by postsurgery status, iv) by metastasis, and v) by recurrence. Salivary LINE1 concentration was highest in the group with recurrence and was significantly higher than that in the control group (Figure 7) $(p=0.020)$. LINE1 concentration in plasma was the highest in the group with metastasis and was significantly higher than that in the control group $(p=0.007)$ (Figure 8).

Correlation between saliva ctDNA and apoptosis in tongue cancer cells. We analyzed the correlation between salivary LINE1 concentration and the apoptosis index, which reflects the degree of apoptosis in tongue cancer, to predict the origin of ctDNA in the saliva. Salivary LINE1 concentration was significantly correlated with the apoptosis index of the tongue ( $\mathrm{r}=0.61, p=0.001)$ (Figure 9).

\section{Discussion}

To the best of our knowledge, this is the first study of the development of an orthotopic HNC animal model for quantitative assessment of salivary ctDNA by human DNA detection in samples.

Derived from all types of apoptotic or necrotic cells, including normal and tumor cells, cell-free DNA (cfDNA) is released into the circulating blood stream or body fluids such as saliva (10). ctDNA can be distinguished from cfDNA by the presence of somatic mutations. Several tumor features, such as stage, angiogenesis and therapeutic response as well as cancer size, are known to be associated with plasma ctDNA concentration (11). ctDNA also provides information on specific tumor- and metastasis-related alterations, such as 

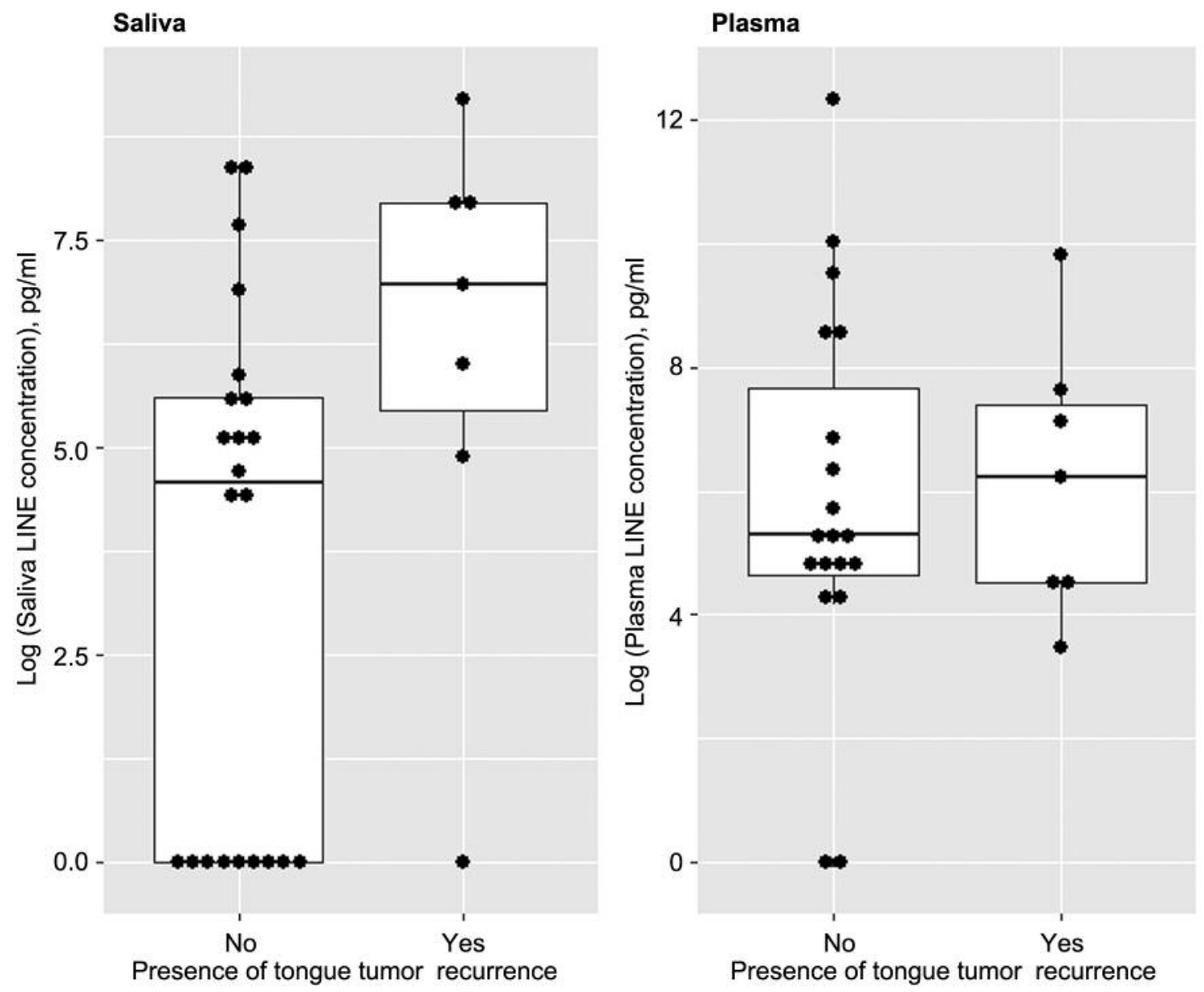

Figure 5. Comparison of long interspersed element-1 (LINE1) concentration of saliva and plasma according to recurrence of tongue tumor.

single-nucleotide mutations, methylation changes, and copynumber variations (12). Significantly, ctDNA has been shown to represent the genome derived from primary tumors and metastases and to reflect the clonal nature of tumor cells $(13,14)$.

Saliva offers a viable alternative to blood samples as a source of DNA for tumor diagnosis and monitoring. Liao et $a l$. found that the amount of DNA from saliva is sufficiently large and of quality suitable to enable PCR amplification, allowing analysis for mutations (15). In our study, we were able to amplify the PCR LINE1 target even in a comparatively small amount of mouse saliva as compared to human samples. Furthermore, the direct contact of cancer cells in the oral cavity may facilitate the detection of tumor DNA, while neovascularization likely happens at a later stage in tumorigenesis, which may explain the low sensitivity of plasma ctDNA (16). ctDNA in saliva not only offers the option of early detection of oral cancer, but also allows simple and less invasive tracking of information about tumor heterogeneity and therapeutic response through serial sampling. However, little is known about the biology of ctDNA in saliva.

Accordingly, we reproduced cancer development, postoperative response, metastasis, and recurrence in an animal model, all of which are clinically important time points in HNC, especially oral cancer. The ctDNA of saliva and plasma at these points was analyzed and the clinical efficacy of the two samples was compared. We attempted to evaluate salivary and plasma ctDNA for monitoring of tumor burden and response to treatment in xenograft models by targeting human-specific DNA sequences. We performed assays using optimal primers reported in previous studies to ensure high sensitivity quantification of human DNA in small amounts of mouse saliva and plasma (8). We measured ctDNA levels through qPCR for detection of LINE1 in orthotopic models. LINE1 is considered to be ideal for quantifying human ctDNA in samples of animal models, based on several studies $(8,17,18)$. 


\section{Saliva}

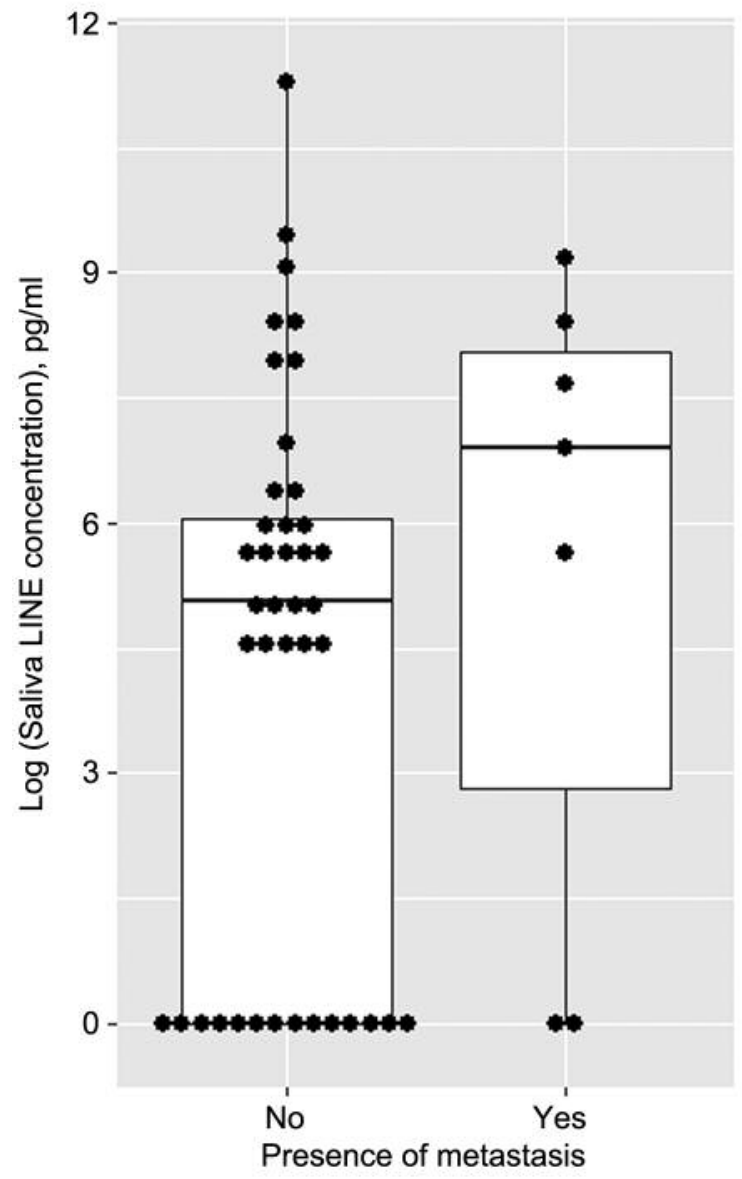

Plasma

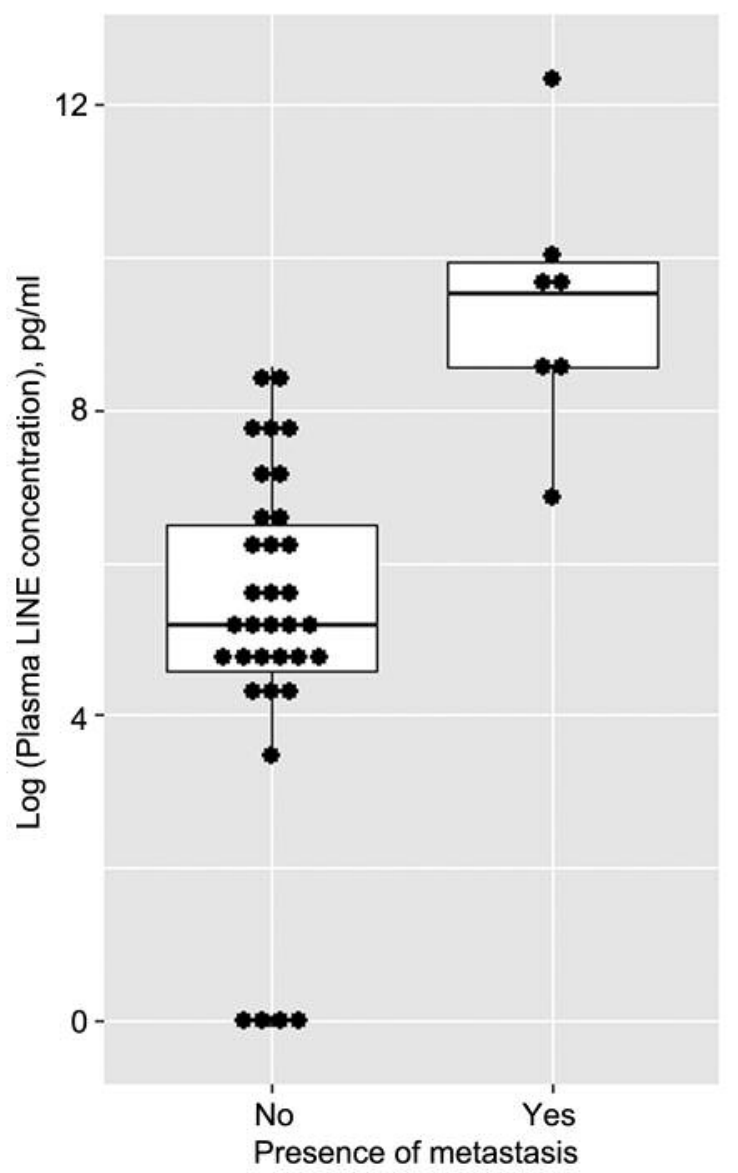

Figure 6. Comparison of long interspersed element-1 (LINE1) concentration of saliva and plasma according to cervical metastasis.

Wang et al. hypothesized that tumor DNA released from the base of HNC epithelial cells to the lymphatic or venous system might be detected in plasma, whereas DNA released on the apical side of HNC would be detectable in the saliva (19). In their results, ctDNA was detected in plasma in $80 \%$ of patients with oral cancer in HNC, while ctDNA was found in $100 \%$ of saliva of patients with oral cancer.

Our animal model was strictly similar to oral cancer because cancer was induced in the tongue. Therefore, in our results, when primary cancer occurred, LINE1 in the saliva was significantly increased, while the level in the plasma was not. Our data indicate that saliva samples give more specific results than plasma samples for ctDNA quantification, at least when originating from an orthotopic HNC model.

It is important to predict cancer development, metastasis, and recurrence in HNC; however, it is difficult to establish an animal model that can reproduce these processes. Muhanna et al. demonstrated the complex kinetic behavior of ctDNA and total plasma DNA upon tumor growth or surgery in a pre- clinical model of HNC (20). However, to our knowledge there has been no study to compare the diagnostic efficacy of salivary ctDNA and plasma ctDNA in detecting metastasis and recurrence after surgery. Disease monitoring and treatment assessment is critical to the clinician in determining subsequent treatment protocols and strategies. Reinert et al. showed the ability of ctDNA monitoring to inform about radicality of the primary resection, response to adjuvant therapy and response to interventions performed in relation to diagnosis of recurrence (21). Tie et al. showed that the presence of ctDNA in a patient's blood after surgery is a sign of a persistent tumor and greatly increased risk of relapse, suggesting that this group of patients may require chemotherapy to prevent recurrence (22). However, in the present study, salivary ctDNA was significantly increased when tongue cancer recurred after surgery, and ctDNA in plasma increased rapidly when describe became metastatic. The release of ctDNA in tumor cells, known to be caused by tumor necrosis or apoptotic cell death, increases when nutrient and oxygen diffusion to cancer tissue is no longer sufficient and 


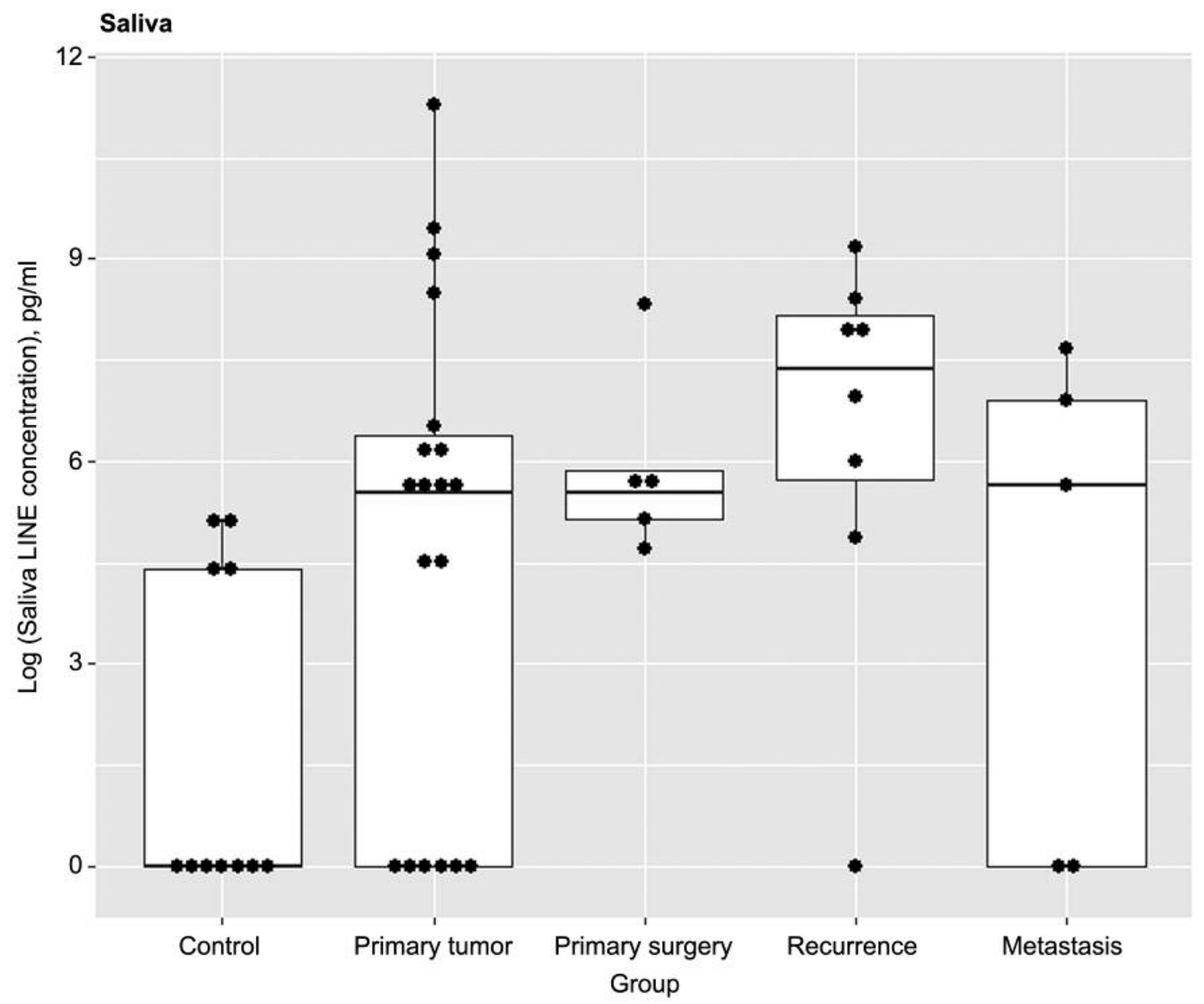

Figure 7. Long interspersed element-1 (LINE1) concentration in saliva among the following groups: i) Control, ii) primary tongue tum or, iii) post surgery, iv) with metastasis, $v$ ) with recurrence.

neovascularization is unsuitable for maintaining the growth of whole tumor cells (23). Therefore, some time is required after the tumor develops until ctDNA can be detected in the bloodstream. Non-blood ctDNA is a fragment of tumor DNA that appears in other body fluids such as urine, saliva, sputum, stool, pleural fluid, and cerebrospinal fluid rather than the plasma (24). Therefore, if saliva is in contact with the free surface of a tumor such as HNC, saliva may be more sensitive than plasma in early detection of recurrence.

The mechanism by which DNA is released into the blood is through apoptosis, necrosis, and DNA release by phagocytes surrounding tumor cells (25). Interestingly, the concentration of LINE1 in saliva was positively correlated with apoptosis index in the pathology of tongue cancer. This finding suggests that the origin of ctDNA in saliva may be HNC and from apoptosis of tumor cells.

This study has some limitations. Firstly, we analyzed saliva and plasma at each time point rather than by serially collecting samples from one individual. In order to evaluate the change in ctDNA according to the change in tumor status, serial sample analysis is ideal, but time point analysis was performed due to a lack of robustness of the animal model. Next, because our findings were from an animal model, validation in real human samples is essential.

In summary, we established an orthotopic HNC mouse model that developed recurrence or metastasis after surgical resection of tumors. In our HNC mouse model, it was possible to assess salivary ctDNA, which reflects tumor development and recurrence. This approach allows for future studies focused on elucidating the mechanisms of production of saliva ctDNA and establishing its effectiveness as a salivary biomarker.

\section{Conflicts of Interest}

None. 


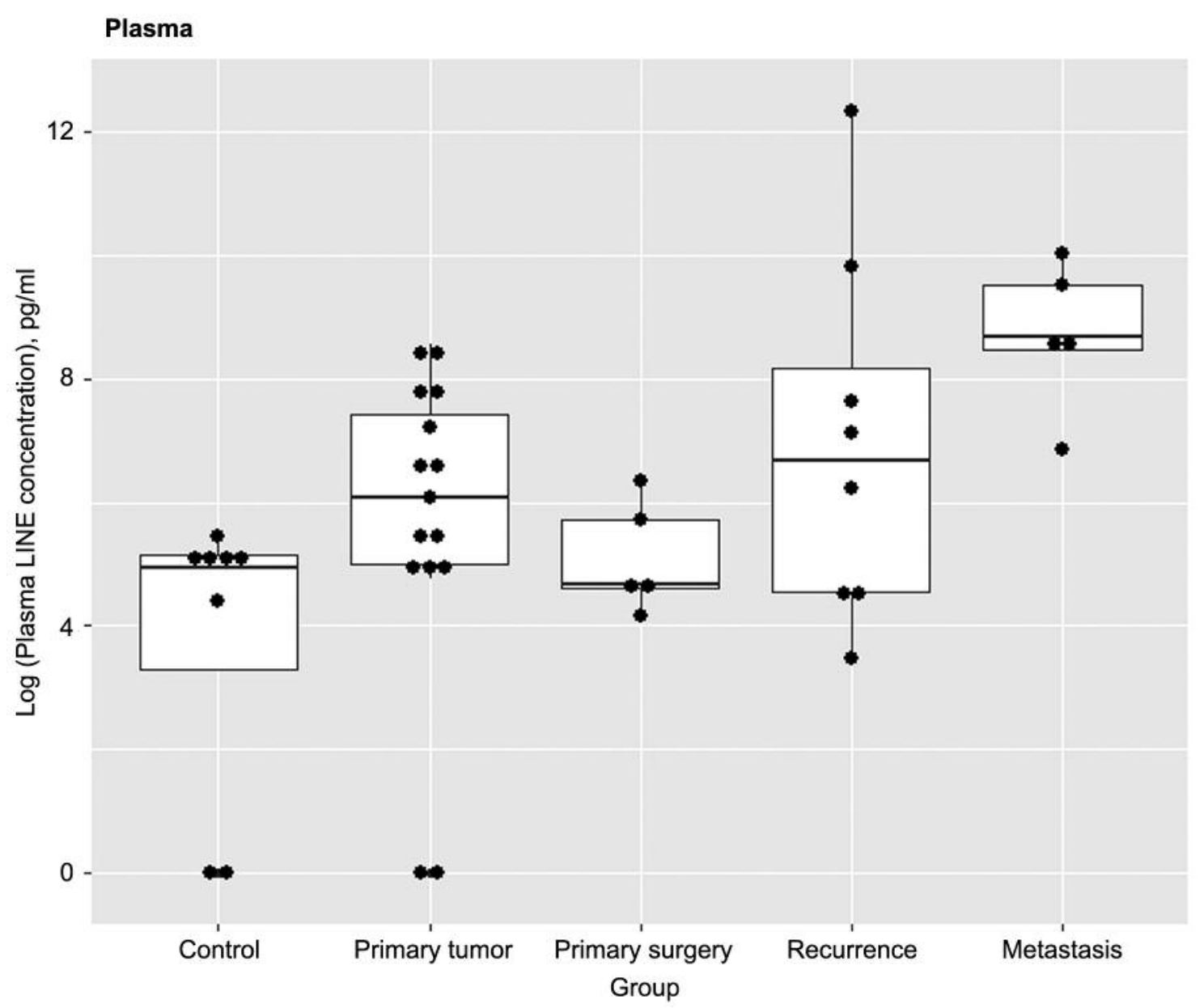

Figure 8. Long interspersed element-1 (LINE1) concentration in plasma among the following groups: i) Control, ii) primary tongue tum or, iii) post surgery, iv) with metastasis, $v$ ) with recurrence.

\section{Authors' Contributions}

Y.C.L, K.Y.W and Y.G.E designed and performed the experiments, analyzed the results, and wrote the article. Y.J.Y. performed the experiments, analyzed the results, and wrote the article. Y.C.L. and Y.G.E conceived the study, designed the experiment, supervised the research, and wrote the article.

\section{Acknowledgements}

This work was supported by a grant from Kyung Hee University in 2019.(KHU-20191221), grant of the Korea Health Technology R\&D Project through the Korea Health Industry Development Institute (KHIDI), funded by the Ministry of Health \& Welfare, Republic of Korea (grant number: HI17C2060), supported by the National Research Foundation of Korea (NRF) Grant funded by the Korea Government (Ministry of Science and ICT) [grant numbers NRF2018R1D1A1B07050154], and supported by Medical Science Research Institute grant, Kyunghee University Hospital at Gangdong in 2018.

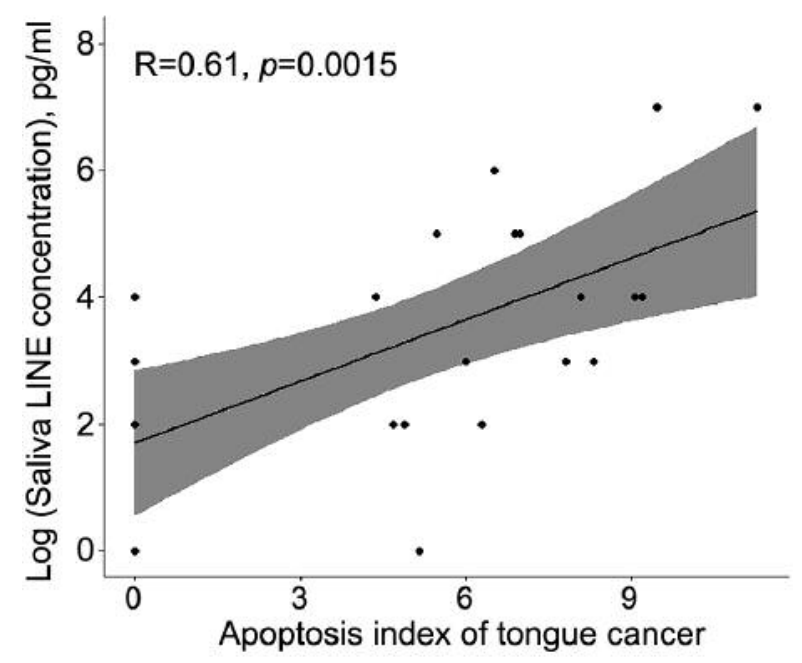

Figure 9. Correlation between salivary long interspersed element-1 (LINE1) concentration and tongue apoptosis index. 


\section{References}

1 de Bree R, van der Putten L, Brouwer J, Castelijns JA, Hoekstra OS and Leemans CR: Detection of locoregional recurrent head and neck cancer after (chemo)radiotherapy using modern imaging. Oral Oncol 45: 386-393, 2009. PMID: 19095487. DOI: 10.1016/j.oraloncology.2008.10.015

2 Müller J, Hüllner M, Strobel K, Huber GF, Burger IA and Haerle SK: The value of 18 F-FDG-PET/CT imaging in oral cavity cancer patients following surgical reconstruction. Laryngoscope 125: 1861-1868, 2015. PMID: 25892275. DOI: 10.1002/lary.25326

3 Zini A, Czerninski R and Sgan-Cohen HD: Oral cancer over four decades: Epidemiology, trends, histology, and survival by anatomical sites. J Oral Pathol Med 39: 299-305, 2010. PMID: 20040019. DOI: 10.1111/j.1600-0714.2009.00845.x

4 Warnakulasuriya S: Global epidemiology of oral and oropharyngeal cancer. Oral Oncol 45: 309-316, 2009. PMID: 18804401. DOI: 10.1016/j.oraloncology.2008.06.002

5 Guerra ENS, Acevedo AC, Leite AF, Gozal D, Chardin H and De Luca Canto G: Diagnostic capability of salivary biomarkers in the assessment of head and neck cancer: A systematic review and meta-analysis. Oral Oncol 51: 805-818, 2015. PMID: 26170140. DOI: 10.1016/j.oraloncology.2015.06.010

6 Sorenson GD, Pribish DM, Valone FH, Memoli VA, Bzik DJ and Yao SL: Soluble normal and mutated DNA sequences from single-copy genes in human blood. Cancer Epidemiol Biomarkers Prev 3: 67-71, 1994. PMID: 8118388.

7 Sano D and Myers JN: Xenograft models of head and neck cancers. Head Neck Oncol 1: 32-32, 2009. PMID: 19678942. DOI: $10.1186 / 1758-3284-1-32$

8 Rago C, Huso DL, Diehl F, Karim B, Liu G, Papadopoulos N, Samuels Y, Velculescu VE, Vogelstein B, Kinzler KW and Diaz LA Jr.: Serial assessment of human tumor burdens in mice by the analysis of circulating DNA. Cancer Res 67: 9364-9370, 2007. PMID: 17909045. DOI: 10.1158/0008-5472.CAN-07-0605

9 Lipponen P, Aaltomaa S, Kosma VM and Syrjänen K: Apoptosis in breast cancer as related to histopathological characteristics and prognosis. Eur J Cancer 30A: 2068-2073, 1994. PMID: 7857705. DOI: 10.1016/0959-8049(94)00342-3

10 van Ginkel JH, Slieker FJB, de Bree R, van Es RJJ, Van Cann EM and Willems SM: Cell-free nucleic acids in body fluids as biomarkers for the prediction and early detection of recurrent head and neck cancer: A systematic review of the literature. Oral Oncol 75: 8-15, 2017. PMID: 29224828. DOI: 10.1016/j.oraloncology. 2017.10.007

11 Chang Y, Tolani B, Nie X, Zhi X, Hu M and He B: Review of the clinical applications and technological advances of circulating tumor DNA in cancer monitoring. Ther Clin Risk Manag 13: 13631374, 2017. PMID: 29066904. DOI: 10.2147/TCRM.S141991

12 Salvi S, Gurioli G, De Giorgi U, Conteduca V, Tedaldi G, Calistri D and Casadio V: Cell-free DNA as a diagnostic marker for cancer: Current insights. Onco Targets Ther 9: 6549-6559, 2016. PMID: 27822059. DOI: 10.2147/OTT.S100901

13 Murtaza M, Dawson S-J, Tsui DWY, Gale D, Forshew T, Piskorz AM, Parkinson C, Chin S-F, Kingsbury Z, Wong ASC, Marass F, Humphray S, Hadfield J, Bentley D, Chin TM, Brenton JD, Caldas $\mathrm{C}$ and Rosenfeld N: Non-invasive analysis of acquired resistance to cancer therapy by sequencing of plasma DNA. Nature 497: 108112, 2013. PMID: 23563269. DOI: 10.1038/nature 12065

14 Alix-Panabières $\mathrm{C}$ and Pantel K: Real-time liquid biopsy: Circulating tumor cells versus circulating tumor DNA. Ann
Transl Med 1: 18-18, 2013. PMID: 25332962. DOI: 10.3978/ j.issn.2305-5839.2013.06.02

15 Liao PH, Chang YC, Huang MF, Tai KW and Chou MY: Mutation of p53 gene codon 63 in saliva as a molecular marker for oral squamous cell carcinomas. Oral Oncol 36: 272-276, 2000. PMID: 10793330. DOI: 10.1016/s1368-8375(00)00005-1

16 Dickinson BT, Kisiel J, Ahlquist DA and Grady WM: Molecular markers for colorectal cancer screening. Gut 64: 1485-1494, 2015. PMID: 25994221. DOI: 10.1136/gutjnl-2014-308075

17 Sunami E, Vu A-T, Nguyen SL, Giuliano AE and Hoon DSB: Quantification of LINE1 in circulating DNA as a molecular biomarker of breast cancer. Ann NY Acad Sci 1137: 171-174, 2008. PMID: 18837943. DOI: 10.1196/annals.1448.011

18 Madhavan D, Wallwiener M, Bents K, Zucknick M, Nees J, Schott S, Cuk K, Riethdorf S, Trumpp A, Pantel K, Sohn C, Schneeweiss A, Surowy $\mathrm{H}$ and Burwinkel B: Plasma DNA integrity as a biomarker for primary and metastatic breast cancer and potential marker for early diagnosis. Breast Cancer Res Treat 146: 163-174, 2014. PMID: 24838941. DOI: 10.1007/s10549-014-2946-2

19 Wang Y, Springer S, Mulvey CL, Silliman N, Schaefer J, Sausen M, James N, Rettig EM, Guo T, Pickering CR, Bishop JA, Chung CH, Califano JA, Eisele DW, Fakhry C, Gourin CG, Ha PK, Kang H, Kiess A, Koch WM, Myers JN, Quon H, Richmon JD, Sidransky D, Tufano RP, Westra WH, Bettegowda C, Diaz LA, Jr., Papadopoulos N, Kinzler KW, Vogelstein B and Agrawal $\mathrm{N}$ : Detection of somatic mutations and HPV in the saliva and plasma of patients with head and neck squamous cell carcinomas. Sci Transl Med 7: 293ra104-293ra104, 2015. PMID: 26109104. DOI: $10.1126 /$ scitranslmed.aaa8507

20 Muhanna N, Di Grappa MA, Chan HHL, Khan T, Jin CS, Zheng Y, Irish JC and Bratman SV: Cell-free DNA kinetics in a preclinical model of head and neck cancer. Sci Rep 7: 16723-16723, 2017. PMID: 29196748. DOI: 10.1038/s41598-017-17079-6

21 Reinert T, Schøler LV, Thomsen R, Tobiasen H, Vang S, Nordentoft I, Lamy P, Kannerup A-S, Mortensen FV, Stribolt K, Hamilton-Dutoit S, Nielsen HJ, Laurberg S, Pallisgaard N, Pedersen JS, Ørntoft TF and Andersen CL: Analysis of circulating tumour DNA to monitor disease burden following colorectal cancer surgery. Gut 65: 625-634, 2016. PMID: 25654990. DOI: 10.1136/gutjnl-2014-308859

22 Tie J, Wang Y, Tomasetti C, Li L, Springer S, Kinde I, Silliman N, Tacey M, Wong H-L, Christie M, Kosmider S, Skinner I, Wong R, Steel M, Tran B, Desai J, Jones I, Haydon A, Hayes T, Price TJ, Strausberg RL, Diaz LA Jr., Papadopoulos N, Kinzler KW, Vogelstein B and Gibbs P: Circulating tumor DNA analysis detects minimal residual disease and predicts recurrence in patients with stage II colon cancer. Sci Transl Med 8: 346ra392-346ra392, 2016. PMID: 27384348. DOI: 10.1126/scitranslmed aaf6219

23 Lo YM, Zhang J, Leung TN, Lau TK, Chang AM and Hjelm NM: Rapid clearance of fetal DNA from maternal plasma. Am J Hum Genet 64: 218-224, 1999. PMID: 9915961. DOI: 10.1086/302205

24 Peng M, Chen C, Hulbert A, Brock MV and Yu F: Non-blood circulating tumor DNA detection in cancer. Oncotarget 8: 6916269173, 2017. PMID: 28978187. DOI: 10.18632/oncotarget.19942

25 Schwarzenbach H, Hoon DSB and Pantel K: Cell-free nucleic acids as biomarkers in cancer patients. Nat Rev Cancer 11: 426437, 2011. PMID: 21562580. DOI: $10.1038 / \mathrm{nrc} 3066$

Received November 13, 2019

Revised November 20, 2019

Accepted November 25, 2019 